\title{
AARP
}

\section{AARP MICHIGAN RETIREMENT SECURITY SURVEY: Annotated Questionnaire}

\section{August 2021}

\section{Screening Questions}

\section{Michigan statewide}

Screening Criteria: Age 25-64, resident of Michigan, registered voter in Michigan, employed or unemployed and looking for work

Telephone $(50 \%$ cell phone $/ 50 \%$ landline)

$\mathrm{n}=\mathbf{6 1 6}$, plus $\mathrm{n}=\mathbf{2 0 0}$ additional African American/Black (AA/B) respondents and $\mathrm{n}=\mathbf{2 0 4}$

Hispanic/Latino $(H / L)$ respondents

Base sample data have been weighted by age, gender, and race/ethnicity according to May 2021 Michigan state voter database statistics. All African American/Black (AA/B) and Hispanic/Latino $(\mathrm{H} / \mathrm{L})$ data have been weighted by age and gender according to May 2021 Michigan state voter database statistics.

S1. [All] Our study is interested in the opinions of certain age groups. Could you please tell me your age as of your last birthday? [IN YEARS] [AGE CODED]

\begin{tabular}{|c|c|c|l|}
\hline $\begin{array}{c}\text { Base \% } \\
(\mathbf{n = 6 1 6})\end{array}$ & $\begin{array}{c}\text { AA/B \% } \\
(\mathbf{n = 2 0 0 )}\end{array}$ & $\begin{array}{c}\mathbf{H} / \mathbf{L} \% \\
(\mathbf{n = 2 0 4})\end{array}$ & \\
\hline 0 & 0 & 0 & Under 25 (TERMINATE) \\
\hline 24 & 28 & 26 & $25-34$ \\
\hline 34 & 35 & 37 & $35-49$ \\
\hline 17 & 16 & 17 & $50-55$ \\
\hline 26 & 21 & 20 & $56-64$ \\
\hline 0 & 0 & 0 & 65 or older (TERMINATE) \\
\hline 0 & 0 & 0 & Refused (TERMINATE) \\
\hline
\end{tabular}

S2. [All] And just to confirm, are you a resident of Michigan?

\begin{tabular}{|c|c|c|l|}
\hline $\begin{array}{c}\text { Base \% } \\
(\mathbf{n = 6 1 6 )}\end{array}$ & $\begin{array}{c}\text { AA/B \% } \\
(\mathbf{n = 2 0 0 )}\end{array}$ & $\begin{array}{c}\mathbf{H} / \mathbf{L} \% \\
(\mathbf{n = 2 0 4})\end{array}$ & \\
\hline 100 & 100 & 100 & Yes \\
\hline 0 & 0 & 0 & No (TERMINATE) \\
\hline 0 & 0 & 0 & Don't know/ refused (TERMINATE) \\
\hline
\end{tabular}


S3. [All] What is your 5-digit ZIP code? [TERMINATE IF NO ONE IN HH IS AGE 25-64 RESIDENT OF MICHIGAN]

S4. [All] And are you registered to vote in Michigan?

\begin{tabular}{|c|c|c|l|}
\hline $\begin{array}{c}\text { Base \% } \\
(\mathbf{n = 6 1 6})\end{array}$ & $\begin{array}{c}\text { AA/B \% } \\
(\mathbf{n = 2 0 0 )}\end{array}$ & $\begin{array}{c}\mathbf{H} / \mathbf{L} \% \\
(\mathbf{n = 2 0 4})\end{array}$ & \\
\hline 100 & 100 & 100 & Yes \\
\hline 0 & 0 & 0 & No (TERMINATE) \\
\hline 0 & 0 & 0 & Don't know (TERMINATE) \\
\hline 0 & 0 & 0 & Refused (TERMINATE) \\
\hline
\end{tabular}

S5. [All] To ensure it is recorded accurately, could you please state your gender?

\begin{tabular}{|c|c|c|l|}
\hline $\begin{array}{c}\text { Base \% } \\
(\mathbf{n = 6 1 6 )}\end{array}$ & $\begin{array}{c}\text { AA/B \% } \\
(\mathbf{n = 2 0 0 )}\end{array}$ & $\begin{array}{c}\mathbf{H} / \mathbf{L} \% \\
(\mathbf{n = 2 0 4 )}\end{array}$ & \\
\hline 49 & 47 & 46 & Male \\
\hline 51 & 53 & 53 & Female \\
\hline 1 & 0 & $<1$ & Other (specify) \\
\hline$<1$ & 0 & 1 & Refused \\
\hline
\end{tabular}

S6. [All] Which of the following best describes your current employment status? Are you...? (READ LIST)

\begin{tabular}{|c|c|c|l|}
\hline $\begin{array}{c}\text { Base } \% \\
(\mathbf{n = 6 1 6})\end{array}$ & $\begin{array}{c}\text { AA/B } \% \\
(\mathbf{n = 2 0 0 )}\end{array}$ & $\begin{array}{c}\mathbf{H} / \mathbf{L} \% \\
\mathbf{( n = 2 0 4 )}\end{array}$ & \\
\hline 14 & 14 & 12 & Self-employed full-time \\
\hline 6 & 6 & 4 & Self-employed part-time \\
\hline 62 & 54 & 68 & Employed full-time \\
\hline 9 & 12 & 6 & Employed part-time \\
\hline 0 & 0 & 0 & Retired and not working at all (TERMINATE) \\
\hline 10 & 15 & 10 & Unemployed and looking for work (SKIP TO Q1) \\
\hline 0 & 0 & 0 & $\begin{array}{l}\text { Or are you not in the labor force for other reasons } \\
\text { (TERMINATE) }\end{array}$ \\
\hline 0 & 0 & 0 & Don't know (VOL) (TERMINATE) \\
\hline 0 & 0 & 0 & Refused (VOL) (TERMINATE) \\
\hline
\end{tabular}

S7. [IF QS6=Employed full- or part-time] Are you currently employed in a federal, state, or local government job?

\begin{tabular}{|c|c|c|l|}
\hline $\begin{array}{c}\text { Base } \% \\
(\mathbf{n}=\mathbf{5 5 7})\end{array}$ & $\begin{array}{c}\text { AA/B \% } \\
(\mathbf{n = 1 7 0})\end{array}$ & $\begin{array}{c}\mathbf{H} / \mathbf{L} \% \\
(\mathbf{n = 1 8 4})\end{array}$ & \\
\hline 0 & 0 & 0 & Yes (TERMINATE) \\
\hline 100 & 100 & 100 & No \\
\hline 0 & 0 & 0 & Don't know (TERMINATE) \\
\hline 0 & 0 & 0 & Refused (TERMINATE) \\
\hline
\end{tabular}




\section{AARP}

\section{Main}

Q1. [All] As you think about your finances in the future, how anxious do you feel about having enough money to live comfortably through your retirement years? Are you...? (READ LIST)

\begin{tabular}{|c|c|c|l|}
\hline $\begin{array}{c}\text { Base \% } \\
(\mathbf{n = 6 1 6 )}\end{array}$ & $\begin{array}{c}\text { AA/B \% } \\
(\mathbf{n = 2 0 0 )}\end{array}$ & $\begin{array}{c}\mathbf{H} / \mathbf{L} \% \\
(\mathbf{n = 2 0 4})\end{array}$ & \\
\hline 23 & 33 & 26 & Very anxious \\
\hline 37 & 38 & 40 & Somewhat anxious \\
\hline 25 & 13 & 20 & Not very anxious \\
\hline 15 & 16 & 13 & Not anxious at all \\
\hline 1 & 1 & 1 & Don't know (VOL) \\
\hline 0 & 0 & 0 & Refused (VOL) \\
\hline
\end{tabular}

Q2_A. [If ages 25-34] Given the amount of money you currently have saved, do you think you'll be able to save enough money for your retirement years?

\begin{tabular}{|c|c|c|l|}
\hline $\begin{array}{c}\text { Base \% } \\
(\mathbf{n}=\mathbf{1 4 8})\end{array}$ & $\begin{array}{c}\text { AA/B \% } \\
(\mathbf{n}=\mathbf{5 5})\end{array}$ & $\begin{array}{c}\mathbf{H} / \mathbf{L} \% \\
(\mathbf{n}=\mathbf{5 2})\end{array}$ & \\
\hline 35 & 38 & 39 & Yes \\
\hline 45 & 47 & 46 & No \\
\hline 20 & 15 & 15 & Don't know \\
\hline 0 & 0 & 0 & Refused \\
\hline
\end{tabular}

Q2_B. [If ages 35-64] Given the amount of money you currently have saved, do you wish you had more money saved for your retirement years?

\begin{tabular}{|c|c|c|l|}
\hline $\begin{array}{c}\text { Base \% } \\
(\mathbf{n = 4 6 8 )}\end{array}$ & $\begin{array}{c}\text { AA/B \% } \\
(\mathbf{n = 1 4 5})\end{array}$ & $\begin{array}{c}\mathbf{H} / \mathbf{L} \% \\
(\mathbf{n}=\mathbf{1 5 2})\end{array}$ & \\
\hline 85 & 92 & 87 & Yes \\
\hline 13 & 7 & 12 & No \\
\hline 2 & 1 & 1 & Don't know \\
\hline 0 & 0 & 0 & Refused \\
\hline
\end{tabular}




\section{AARP}

Q3. [All] How confident are you that you will have enough money to take care of your healthcare expenses during your retirement years? These expenses include things such as co-payments, deductibles, out-of-pocket drug costs, expenses that Medicare doesn't cover, such as hearing aids and eyeglasses, and possibly nursing home or long-term care. Are you...? (READ LIST)

\begin{tabular}{|c|c|c|l|}
\hline $\begin{array}{c}\text { Base \% } \\
(\mathbf{n = 6 1 6 )}\end{array}$ & $\begin{array}{c}\text { AA/B \% } \\
(\mathbf{n = 2 0 0 )}\end{array}$ & $\begin{array}{c}\mathbf{H} / \mathbf{L} \% \\
(\mathbf{n = 2 0 4 )}\end{array}$ & \\
\hline 16 & 18 & 17 & Very confident \\
\hline 38 & 31 & 28 & Somewhat confident \\
\hline 24 & 28 & 27 & Not very confident \\
\hline 18 & 18 & 25 & Not confident at all \\
\hline 3 & 4 & 3 & Don't know (VOL) \\
\hline 0 & 1 & $<1$ & Refused (VOL) \\
\hline
\end{tabular}

Q4. [All] How concerned are you that cost-of-living increases (over which you have no control) may reduce your standard of living? Are you...? (READ LIST)

\begin{tabular}{|c|c|c|l|}
\hline $\begin{array}{c}\text { Base \% } \\
(\mathbf{n = 6 1 6 )}\end{array}$ & $\begin{array}{c}\text { AA/B \% } \\
(\mathbf{n = 4 0 )}\end{array}$ & $\begin{array}{c}\mathbf{H} / \mathbf{L} \% \\
(\mathbf{n = 2 0 4})\end{array}$ & \\
\hline 32 & 40 & 37 & Very concerned \\
\hline 43 & 35 & 38 & Somewhat concerned \\
\hline 16 & 12 & 15 & Not very concerned \\
\hline 8 & 11 & 7 & Not concerned at all \\
\hline 1 & 3 & 2 & Don't know (VOL) \\
\hline 0 & 0 & 1 & Refused (VOL) \\
\hline
\end{tabular}

Q5. [All] How important is it for people to be able to save money for their retirement years while they are working? (READ LIST)

\begin{tabular}{|c|c|c|l|}
\hline $\begin{array}{c}\text { Base \% } \\
(\mathbf{n = 6 1 6 )}\end{array}$ & $\begin{array}{c}\text { AA/B \% } \\
(\mathbf{n = 2 0 0 )}\end{array}$ & $\begin{array}{c}\mathbf{H} / \mathbf{L} \% \\
(\mathbf{n = 2 0 4 )}\end{array}$ & \\
\hline 86 & 84 & 82 & Very important \\
\hline 11 & 11 & 16 & Somewhat important \\
\hline 2 & 2 & $<1$ & Not very important \\
\hline$<1$ & 2 & 1 & Not important at all \\
\hline 1 & 1 & $<1$ & Don't know (VOL) \\
\hline$<1$ & 0 & $<1$ & Refused (VOL) \\
\hline
\end{tabular}

Q6. [All] When it comes to planning and saving for retirement, would you say that you are ahead of schedule, on track, or behind schedule?

\begin{tabular}{|c|c|c|l|}
\hline $\begin{array}{c}\text { Base \% } \\
(\mathbf{n = 6 1 6 )}\end{array}$ & $\begin{array}{c}\text { AA/B \% } \\
(\mathbf{n = 2 0 0 )}\end{array}$ & $\begin{array}{c}\mathbf{H} / \mathbf{L} \% \\
(\mathbf{n = 2 0 4})\end{array}$ & \\
\hline 9 & 7 & 7 & Ahead of schedule \\
\hline 39 & 32 & 32 & On track \\
\hline 50 & 55 & 56 & Behind schedule \\
\hline 3 & 7 & 4 & Don't know \\
\hline 0 & 0 & 0 & Refused \\
\hline
\end{tabular}




\section{AARP}

Q7. [If S6=Employed full- or part-time] Which of the following ways to save for retirement does your current employer provide? Do they provide...? (READ LIST) [ROTATE A-B/B-A]

\begin{tabular}{|c|c|c|l|}
\hline $\begin{array}{c}\text { Base \% } \\
(\mathbf{n}=\mathbf{4 3 6})\end{array}$ & $\begin{array}{c}\text { AA/B \% } \\
(\mathbf{n}=131)\end{array}$ & $\begin{array}{c}\mathbf{H} / \mathbf{L} \% \\
(\mathbf{n}=151)\end{array}$ & \\
\hline 21 & 32 & 16 & $\begin{array}{l}\text { A. A traditional pension plan or a defined benefit plan? [IF } \\
\text { ASKED: "A defined benefit plan supplies retirees with a } \\
\text { monthly income, typically based on a formula of salary and } \\
\text { years of service. It is not a 401(k) plan."] }\end{array}$ \\
\hline 71 & 63 & 74 & $\begin{array}{l}\text { B. An IRA, 401(k) or 403(b) defined contribution plan? [IF } \\
\text { ASKED: “A defined contribution plan allows you to make } \\
\text { contributions from your salary to an individual account set up in } \\
\text { your name.'] }\end{array}$ \\
\hline 17 & 15 & 19 & $\begin{array}{l}\text { C. (If neither above=yes, confirm:) Your employer does NOT } \\
\text { offer a way to save for retirement. }\end{array}$ \\
\hline 3 & 7 & 2 & Don't know (VOL) \\
\hline 1 & 3 & 1 & Refused (VOL) \\
\hline
\end{tabular}

Q7_A. [If Q7=Your employer does NOT offer a way to save for retirement, don't know, or refused] If your employer offered a way to save for retirement at work, how likely would you be to take advantage of it? (READ LIST)

\begin{tabular}{|c|c|c|l|}
\hline $\begin{array}{c}\text { Base \% } \\
(\mathbf{n = 9 0})^{*}\end{array}$ & $\begin{array}{c}\text { AA/B \% } \\
(\mathbf{n = 3 3})^{*}\end{array}$ & $\begin{array}{c}\mathbf{H} / \mathbf{L} \% \\
(\mathbf{n = 3 3})^{*}\end{array}$ & \\
\hline 65 & 72 & 69 & Very likely \\
\hline 15 & 11 & 17 & Somewhat likely \\
\hline 5 & 0 & 4 & Not very likely \\
\hline 5 & 6 & 4 & Not at all likely \\
\hline 8 & 5 & 3 & Don't know (VOL) \\
\hline 2 & 6 & 2 & Refused (VOL) \\
\hline
\end{tabular}

${ }^{\star}$ Note: Small base. Use caution when generalizing to the larger statewide, African American/Black, and Hispanic/Latino populations. 


\section{AARP}

Q8. Are you making regular contributions to any of the following savings tools...? (READ LIST) [ROTATE A-B/B-A, READ C LAST]

\begin{tabular}{|l|c|c|c|c|}
\hline & $\begin{array}{c}\text { Yes } \\
\%\end{array}$ & $\begin{array}{c}\text { No } \\
\%\end{array}$ & $\begin{array}{c}\text { Not } \\
\text { sure } \\
\%\end{array}$ & $\begin{array}{c}\text { Refused } \\
\%\end{array}$ \\
\hline $\begin{array}{l}\text { A. [If Q7=An IRA, 401(k) or 403(b) defined contribution plan] A workplace retirement saving } \\
\text { plan such as a 401(k) or 403(b) offered by your employer }\end{array}$ \\
\hline Base \% (n=309) & 89 & 11 & 1 & 0 \\
\hline AA/B \% (n=82) & 92 & 7 & 0 & 1 \\
\hline H/L \% (n=112) & 90 & 9 & 0 & 1 \\
\hline B. [All] A personal retirement savings plan such as an IRA or thrift savings plan & 3 & 1 \\
\hline Base \% (n=616) & 41 & 55 & 2 \\
\hline AA/B \% (n=200) & 35 & 56 & 7 & 2 \\
\hline H/L \% (n=204) & 38 & 58 & 5 & 0 \\
\hline C. [All] Something else to help you save for retirement & 40 & 55 & 4 & $<1$ \\
\hline Base \% (n=616) & 37 & 55 & 5 & 2 \\
\hline AA/B \% (n=200) & 44 & 48 & 7 & $<1$ \\
\hline H/L \% (n=204)
\end{tabular}

Q9. [All] How concerned are you as a taxpayer that some Michigan workers have not saved enough money for retirement and could end up being reliant on public assistance programs? Are you...? (READ LIST)

\begin{tabular}{|c|c|c|l|}
\hline $\begin{array}{c}\text { Base \% } \\
(\mathbf{n = 6 1 6 )}\end{array}$ & $\begin{array}{c}\text { AA/B \% } \\
(\mathbf{n = 2 0 0 )}\end{array}$ & $\begin{array}{c}\mathbf{H} / \mathbf{L} \% \\
(\mathbf{n = 2 0 4 )}\end{array}$ & \\
\hline 32 & 39 & 37 & Very concerned \\
\hline 42 & 32 & 40 & Somewhat concerned \\
\hline 13 & 14 & 14 & Not very concerned \\
\hline 11 & 13 & 6 & Not concerned at all \\
\hline 2 & 2 & 3 & Not sure/ Don't know (VOL) \\
\hline$<1$ & 0 & 0 & Refused (VOL) \\
\hline
\end{tabular}




\section{AARP'}

Thank you. Now, I just have a few questions on how the Coronavirus pandemic has impacted your finances.

Q10. [All] Has your household income declined since the coronavirus outbreak?

\begin{tabular}{|c|c|c|l|}
\hline $\begin{array}{c}\text { Base \% } \\
(\mathbf{n}=616)\end{array}$ & $\begin{array}{c}\text { AA/B \% } \\
(\mathbf{n}=\mathbf{2 0 0})\end{array}$ & $\begin{array}{c}\mathbf{H} / \mathbf{L} \% \\
(\mathbf{n = 2 0 4})\end{array}$ & \\
\hline $\mathbf{4 2}$ & 47 & 46 & Yes \\
\hline $\mathbf{5 8}$ & 53 & 54 & No \\
\hline $\mathbf{0}$ & 0 & 0 & Don't know \\
\hline $\mathbf{0}$ & 0 & 0 & Refused \\
\hline
\end{tabular}

Q11. [lf employed or self-employed full- or part-time or unemployed and looking for work] At any time since March of 2020, were you or anyone in your household laid off or furloughed from a job because of the coronavirus outbreak?

\begin{tabular}{|c|c|c|l|}
\hline $\begin{array}{c}\text { Base \% } \\
(\mathbf{n}=\mathbf{6 1 6})\end{array}$ & $\begin{array}{c}\mathbf{A A} / \mathbf{B} \% \\
(\mathbf{n}=\mathbf{2 0 0})\end{array}$ & $\begin{array}{c}\mathbf{H} / \mathbf{L} \% \\
(\mathbf{n = 2 0 4 )}\end{array}$ & \\
\hline $\mathbf{4 6}$ & 52 & 53 & Yes \\
\hline $\mathbf{5 3}$ & 47 & 47 & No \\
\hline $\mathbf{1}$ & 2 & 0 & Don't know \\
\hline $\mathbf{0}$ & 0 & 0 & Refused \\
\hline
\end{tabular}

Q12. [All] And at any time since March of 2020, have you or anyone in your household had to take a cut in pay at work due to reduced hours?

\begin{tabular}{|c|c|c|l|}
\hline $\begin{array}{c}\text { Base \% } \\
(\mathbf{n = 6 1 6})\end{array}$ & $\begin{array}{c}\text { AA/B \% } \\
(\mathbf{n}=\mathbf{2 0 0})\end{array}$ & $\begin{array}{c}\mathbf{H} / \mathbf{L} \% \\
(\mathbf{n = 2 0 4 )}\end{array}$ & \\
\hline 37 & 44 & 42 & Yes \\
\hline 62 & 55 & 57 & No \\
\hline 1 & 1 & $<1$ & Don't know \\
\hline 0 & 0 & 1 & Refused \\
\hline
\end{tabular}




\section{AARP'}

Q13. [If any of $\mathrm{Q} 8=Y e s]$ Prior to the coronavirus outbreak, approximately what percent of your total income were you saving on average each month in retirement plans, such as a $401 \mathrm{k}$, IRA, or another retirement plan? [READ LIST]

\begin{tabular}{|c|c|c|l|}
\hline $\begin{array}{c}\text { Base \% } \\
(\mathbf{n = 4 6 9 )}\end{array}$ & $\begin{array}{c}\text { AA/B \% } \\
(\mathbf{n = 1 4 0 )}\end{array}$ & $\begin{array}{c}\mathbf{H} / \mathbf{L} \% \\
(\mathbf{n = 1 6 0 )}\end{array}$ & \\
\hline 4 & 5 & 3 & $0 \%$ \\
\hline 21 & 25 & 21 & $1 \%$ to $5 \%$ \\
\hline 33 & 28 & 31 & $6 \%$ to $10 \%$ \\
\hline 18 & 16 & 17 & $11 \%$ to $15 \%$ \\
\hline 12 & 9 & 10 & $16 \%$ to $20 \%$ \\
\hline 6 & 10 & 7 & More than $20 \%$ \\
\hline 5 & 8 & 10 & Don't know (VOL) \\
\hline 1 & 1 & 1 & Refused (VOL) \\
\hline
\end{tabular}

Q14. [If any of Q8=Yes] Since the coronavirus outbreak, approximately what percent of your total income are you saving now in retirement plans, such as a $401 \mathrm{k}$, IRA, or another retirement plan? [READ LIST]

\begin{tabular}{|c|c|c|l|}
\hline $\begin{array}{c}\text { Base \% } \\
(\mathbf{n = 4 6 9 )}\end{array}$ & $\begin{array}{c}\text { AA/B \% } \\
(\mathbf{n = 1 4 0 )}\end{array}$ & $\begin{array}{c}\mathbf{H} / \mathbf{L} \% \\
(\mathbf{n = 1 6 0 )}\end{array}$ & \\
\hline 12 & 14 & 12 & $0 \%$ \\
\hline 22 & 22 & 20 & $1 \%$ to $5 \%$ \\
\hline 27 & 28 & 28 & $6 \%$ to $10 \%$ \\
\hline 19 & 10 & 16 & $11 \%$ to $15 \%$ \\
\hline 12 & 10 & 11 & $16 \%$ to $20 \%$ \\
\hline 4 & 8 & 4 & More than $20 \%$ \\
\hline 4 & 7 & 9 & Don't know (VOL) \\
\hline 0 & 1 & 1 & Refused (VOL) \\
\hline
\end{tabular}

Q15. [All] Have your attitudes about saving for your retirement changed since the coronavirus outbreak in the U.S.?

\begin{tabular}{|c|c|c|l|}
\hline $\begin{array}{c}\text { Base \% } \\
(\mathbf{n = 6 1 6 )}\end{array}$ & $\begin{array}{c}\text { AA/B \% } \\
(\mathbf{n = 2 0 0 )}\end{array}$ & $\begin{array}{c}\mathbf{H} / \mathbf{L} \% \\
(\mathbf{n = 2 0 4 )}\end{array}$ & \\
\hline 29 & 49 & 40 & Yes \\
\hline 66 & 46 & 51 & No \\
\hline 5 & 5 & 9 & Don't know \\
\hline$<1$ & 1 & 0 & Refused \\
\hline
\end{tabular}




\section{AARP}

Q16. [IF Q8a/b/c=YES] What actions have you taken since the coronavirus outbreak in the U.S. in regard to your savings in retirement plan(s)? [READ LIST; ACCEPT MULTIPLE RESPONSES EXCEPT FOR 1-2 (MUTUALLY EXCLUSIVE - CANNOT SELECT BOTH AND 8 (CANNOT SELECT 8 AND ANY OTHER OPTIONS] [RANDOMIZE ORDER OF 1-6

\begin{tabular}{|c|c|c|l|}
\hline $\begin{array}{c}\text { Base \% } \\
(\mathbf{n}=\mathbf{4 6 9 )}\end{array}$ & $\begin{array}{c}\text { AA/B \% } \\
(\mathbf{n}=\mathbf{1 4 0})\end{array}$ & $\begin{array}{c}\mathbf{H} / \mathbf{L} \% \\
\mathbf{( n = 1 6 0 )}\end{array}$ & \\
\hline 17 & 9 & 13 & Re-allocated money to different investment(s) within your plan \\
\hline 14 & 15 & 8 & Reduced the amount you are contributing \\
\hline 10 & 14 & 14 & Withdrew some money early \\
\hline 7 & 7 & 7 & Are no longer contributing \\
\hline 5 & 6 & 5 & $\begin{array}{l}\text { Took a loan against your 401(k) or other retirement savings } \\
\text { account }\end{array}$ \\
\hline 3 & 7 & 1 & Withdrew all money early \\
\hline 3 & 3 & 2 & Other (specify) \\
\hline 51 & 51 & 51 & You have not taken any action with your retirement savings \\
\hline 2 & 2 & 4 & Don't know or refused (VOL) \\
\hline
\end{tabular}

Q17. [AIl] What financial steps, if any, are you taking as a result of the coronavirus outbreak? [READ LIST, ALLOW MULTIPLE RESPONSES FOR 1-8; RANDOMIZE ORDER OF 1-7]

\begin{tabular}{|c|c|c|l|}
\hline $\begin{array}{c}\text { Base \% } \\
(\mathbf{n = 6 1 6})\end{array}$ & $\begin{array}{c}\text { AA/B \% } \\
(\mathbf{n = 2 0 0 )}\end{array}$ & $\begin{array}{c}\mathbf{H} / \mathbf{L} \% \\
(\mathbf{n = 2 0 4 )}\end{array}$ & \\
\hline 49 & 46 & 48 & Reducing nonessential spending \\
\hline 30 & 36 & 29 & Building up an emergency savings fund \\
\hline 30 & 34 & 29 & $\begin{array}{l}\text { Postponing large financial decisions (e.g. buying a house, } \\
\text { furniture, car) }\end{array}$ \\
\hline 25 & 24 & 28 & $\begin{array}{l}\text { Finding more affordable options (e.g. health, car, or life } \\
\text { insurance) }\end{array}$ \\
\hline 13 & 12 & 17 & $\begin{array}{l}\text { Postponing payments to companies or lenders who are offering } \\
\text { a grace period or relief }\end{array}$ \\
\hline 11 & 12 & 10 & Buying more items on credit \\
\hline 10 & 9 & 11 & $\begin{array}{l}\text { Reallocating what would have been savings for retirement to } \\
\text { an emergency fund }\end{array}$ \\
\hline 2 & 1 & 1 & Other (specify) \\
\hline 24 & 19 & 21 & None of the above \\
\hline 1 & 3 & 3 & Don't know or refused (VOL) \\
\hline
\end{tabular}




\section{AARP}

Thank you. Now, I have some questions to ask about a retirement savings program for Michigan residents.

SW1. [AIl] Many in Michigan, especially those who work for small businesses, do not have a way to save for retirement at work. One way to help more workers save would be for Michigan to set up a retirement savings program. The program offers retirement savings workers control by allowing them to choose whether or not to participate, decide how much to contribute, and let them take their account with them if they change jobs. Workers save for retirement using money automatically taken out of their regular paycheck. The program would be managed as a public-private partnership. Do you support or oppose this program? Is that strongly or somewhat?

\begin{tabular}{|c|c|c|l|}
\hline $\begin{array}{c}\text { Base \% } \\
(\mathbf{n = 6 1 6 )}\end{array}$ & $\begin{array}{c}\text { AA/B \% } \\
(\mathbf{n = 2 0 0 )}\end{array}$ & $\begin{array}{c}\mathbf{H} / \mathbf{L} \% \\
(\mathbf{n = 2 0 4})\end{array}$ & \\
\hline 29 & 39 & 26 & Strongly support \\
\hline 36 & 31 & 38 & Somewhat support \\
\hline 14 & 16 & 13 & Neither support nor oppose \\
\hline 6 & 4 & 3 & Somewhat oppose \\
\hline 10 & 3 & 7 & Strongly oppose \\
\hline 5 & 7 & 13 & Don't know \\
\hline 1 & 0 & 1 & Refused \\
\hline
\end{tabular}

SW2. [AIl] Now I'm going to read a list of some features that could be considered for a Michigan retirement savings program and l'd like you to tell me how important each would be to you. After I finish reading each statement, tell me if that feature is very important to you, somewhat important, not very important, or not at all important to you. How important is it to you that a state savings program be...? (READ LIST)

\begin{tabular}{|c|c|c|c|c|c|c|}
\hline & $\begin{array}{c}\text { Very } \\
\text { important } \\
\%\end{array}$ & $\begin{array}{c}\text { Somewhat } \\
\text { important } \\
\%\end{array}$ & $\begin{array}{c}\text { Not very } \\
\text { important } \\
\%\end{array}$ & $\begin{array}{c}\text { Not } \\
\text { important } \\
\text { at all } \\
\%\end{array}$ & $\begin{array}{c}\text { Don't } \\
\text { know } \\
\text { (VOL) } \\
\%\end{array}$ & $\begin{array}{c}\text { Refused } \\
\text { (VOL) } \\
\%\end{array}$ \\
\hline
\end{tabular}

A. Portable, so if you leave one job, you can take the money and account with you to your next job

\begin{tabular}{|l|l|l|l|l|l|l|}
\hline Base \% (n=616) & 76 & 12 & 4 & 5 & 3 & 1 \\
\hline AA/B \% (n=200) & 75 & 18 & 3 & 3 & 1 & 0 \\
\hline H/L \% (n=204) & 76 & 15 & 4 & 2 & 3 & 1 \\
\hline
\end{tabular}

B. Pre-taxed, contributions are automatically taken from each paycheck before taxes

\begin{tabular}{|l|l|l|l|l|l|l|}
\hline Base \% (n=616) & 50 & 32 & 6 & 8 & 3 & 1 \\
\hline AA/B \% (n=200) & 53 & 30 & 8 & 3 & 4 & 2 \\
\hline H/L \% (n=204) & 49 & 33 & 8 & 5 & 6 & 0 \\
\hline
\end{tabular}

C. Voluntary, so participants are not required to make contributions

\begin{tabular}{|l|l|l|l|l|l|l|}
\hline Base \% (n=616) & 61 & 23 & 6 & 7 & 3 & 1 \\
\hline AA/B \% (n=200) & 55 & 24 & 10 & 6 & 5 & 1 \\
\hline H/L \% (n=204) & 61 & 29 & 4 & 3 & 4 & $<1$ \\
\hline
\end{tabular}

D. Available to all employees who do not have a way to save for retirement at work, including those who work for small businesses

\begin{tabular}{|l|l|l|l|l|l|l|}
\hline Base \% (n=616) & 65 & 22 & 4 & 6 & 3 & 1 \\
\hline AA/B \% (n=200) & 71 & 16 & 5 & 3 & 5 & 0 \\
\hline H/L \% (n=204) & 68 & 19 & 5 & 6 & 3 & 0 \\
\hline
\end{tabular}




\section{AARP'}

SW3. [All] Americans are more likely to save for retirement when they can do so out of their regular paycheck at work. Yet many workers do not have access to a workplace retirement savings plan, especially those that work at small businesses. Thinking about this, do you agree or disagree with the following statement: "Michigan policymakers should make it easier for all workers to save for retirement out of their regular paycheck." Do you agree or disagree with this statement? And is that strongly or somewhat?

\begin{tabular}{|c|c|c|l|}
\hline $\begin{array}{c}\text { Base \% } \\
(\mathbf{n = 6 1 6 )}\end{array}$ & $\begin{array}{c}\text { AA/B \% } \\
(\mathbf{n = 2 0 0 )}\end{array}$ & $\begin{array}{c}\mathbf{H} / \mathbf{L} \% \\
(\mathbf{n = 2 0 4})\end{array}$ & \\
\hline 57 & 72 & 62 & Strongly agree \\
\hline 26 & 16 & 24 & Somewhat agree \\
\hline 7 & 5 & 6 & Neither agree nor disagree \\
\hline 4 & 1 & 3 & Somewhat disagree \\
\hline 5 & 5 & 3 & Strongly disagree \\
\hline 1 & 2 & 3 & Don't know \\
\hline$<1$ & 0 & 0 & Refused \\
\hline
\end{tabular}

Q18. [AIl] Thinking about the many concerns and issues that workers and employers are facing today related to the impact of the COVID-19 pandemic, do you agree or disagree that Michigan policymakers should make it easier for employees of small businesses and sole business owners to save for retirement? (Is that strongly or somewhat?)

\begin{tabular}{|c|c|c|l|}
\hline $\begin{array}{c}\text { Base \% } \\
(\mathbf{n = 6 1 6 )}\end{array}$ & $\begin{array}{c}\text { AA/B \% } \\
(\mathbf{n = 2 0 0 )}\end{array}$ & $\begin{array}{c}\mathbf{H} / \mathbf{L} \% \\
(\mathbf{n = 2 0 4})\end{array}$ & \\
\hline 57 & 70 & 58 & Strongly agree \\
\hline 27 & 17 & 21 & Somewhat agree \\
\hline 6 & 7 & 13 & Neither agree nor disagree (VOL) \\
\hline 3 & 3 & 3 & Somewhat disagree \\
\hline 5 & 1 & 3 & Strongly disagree \\
\hline 2 & 4 & 2 & Don't know (VOL) \\
\hline$<1$ & 0 & 0 & Refused (VOL) \\
\hline
\end{tabular}

The following questions are for classification purposes only and will be kept entirely confidential.

D1. [All] Do you do something to earn extra money that is not your main source of income?

\begin{tabular}{|c|c|c|l|}
\hline $\begin{array}{c}\text { Base \% } \\
(\mathbf{n = 6 1 6 )}\end{array}$ & $\begin{array}{c}\text { AA/B \% } \\
(\mathbf{n = 2 0 0 )}\end{array}$ & $\begin{array}{c}\mathbf{H} / \mathbf{L} \% \\
(\mathbf{n = 2 0 4 )}\end{array}$ & \\
\hline 42 & 36 & 36 & Yes \\
\hline 56 & 63 & 61 & No \\
\hline 2 & 1 & 3 & Don't know \\
\hline$<1$ & $<1$ & $<1$ & Refused \\
\hline
\end{tabular}




\section{AARP}

D2. [All] What is the highest level of education that you completed? (READ LIST)

\begin{tabular}{|c|c|c|l|}
\hline $\begin{array}{c}\text { Base \% } \\
(\mathbf{n = 6 1 6 )}\end{array}$ & $\begin{array}{c}\text { AA/B \% } \\
(\mathbf{n = 2 0 0 )}\end{array}$ & $\begin{array}{c}\mathbf{H} / \mathbf{L} \% \\
(\mathbf{n = 2 0 4 )}\end{array}$ & \\
\hline 1 & 4 & 2 & 0-12th grade (no diploma) \\
\hline 15 & 17 & 18 & High school graduate (or equivalent) \\
\hline 17 & 17 & 20 & Post-high school education (no degree) \\
\hline 15 & 17 & 16 & 2-year college degree \\
\hline 28 & 23 & 24 & 4-year college degree \\
\hline 4 & 4 & 4 & Post-graduate study (no degree) \\
\hline 19 & 17 & 15 & Graduate or professional degree \\
\hline 1 & 1 & 1 & Don't know (VOL) \\
\hline$<1$ & 0 & 0 & Refused (VOL) \\
\hline
\end{tabular}

D3. [AIl] What is your current marital status? Are you currently...? (READ LIST)

\begin{tabular}{|c|c|c|l|}
\hline $\begin{array}{c}\text { Base \% } \\
(\mathbf{n = 6 1 6 )}\end{array}$ & $\begin{array}{c}\text { AA/B \% } \\
(\mathbf{n = 2 0 0 )}\end{array}$ & $\begin{array}{c}\text { H/L \% } \\
(\mathbf{n}=\mathbf{2 0 4})\end{array}$ & \\
\hline 63 & 44 & 67 & Married \\
\hline 7 & 8 & 8 & Not married, living with your partner \\
\hline 2 & 2 & 2 & Separated \\
\hline 8 & 4 & 9 & Divorced \\
\hline 2 & 1 & 0 & Widowed \\
\hline 18 & 40 & 14 & Single and never been married \\
\hline$<1$ & 0 & 1 & Not sure/ Don't know (VOL) \\
\hline 1 & $<1$ & 0 & Refused (VOL) \\
\hline
\end{tabular}

D4. [If D3=Married or not married living with your partner and ages 40-64] Are you or your (spouse/partner) currently a member of A-A-R-P? [If D3=Separated, divorced, widowed, single and never been married, don't know, or refused and ages 50-64] Are you currently a member of A-A-R$\mathrm{P}$ ?

\begin{tabular}{|c|c|c|l|}
\hline $\begin{array}{c}\text { Base \% } \\
(\mathbf{n = 3 5 8})\end{array}$ & $\begin{array}{c}\text { AA/B \% } \\
(\mathbf{n}=\mathbf{9 7})\end{array}$ & $\begin{array}{c}\mathbf{H} / \mathbf{L} \% \\
(\mathbf{n = 1 1 4})\end{array}$ & \\
\hline 18 & 28 & 14 & Yes \\
\hline 81 & 70 & 82 & No \\
\hline 1 & 2 & 3 & Don't know \\
\hline 0 & 0 & 1 & Refused \\
\hline
\end{tabular}

D5. [All] Are you of Hispanic, Spanish, or Latino origin or descent?

\begin{tabular}{|c|c|c|l|}
\hline $\begin{array}{c}\text { Base \% } \\
(\mathbf{n = 6 1 6})\end{array}$ & $\begin{array}{c}\text { AA/B \% } \\
(\mathbf{n = 2 0 0 )}\end{array}$ & $\begin{array}{c}\mathbf{H} / \mathbf{L} \% \\
(\mathbf{n}=\mathbf{2 0 4})\end{array}$ & \\
\hline 3 & 0 & 100 & Yes \\
\hline 95 & 100 & 0 & No \\
\hline 1 & 0 & 0 & Don't know \\
\hline 1 & 1 & 0 & Refused \\
\hline
\end{tabular}




\section{AARP}

D6. [All] What is your race? Are you...? (READ LIST)

\begin{tabular}{|c|c|c|l|}
\hline $\begin{array}{c}\text { Base \% } \\
(\mathbf{n = 6 1 6 )}\end{array}$ & $\begin{array}{c}\text { AA/B \% } \\
(\mathbf{n = 2 0 0 )}\end{array}$ & $\begin{array}{c}\mathbf{H} / \mathbf{L} \% \\
(\mathbf{n = 2 0 4})\end{array}$ & \\
\hline 84 & 0 & 71 & White or Caucasian \\
\hline 10 & 100 & 12 & Black or African American \\
\hline 1 & 0 & 0 & Asian \\
\hline 1 & 0 & 0 & American Indian or Alaska Native \\
\hline 0 & 0 & 1 & Native Hawaiian or other Pacific Islander \\
\hline 1 & 0 & 11 & Other (specify) \\
\hline 0 & 0 & 2 & Don't know (VOL) \\
\hline 2 & 0 & 4 & Refused (VOL) \\
\hline
\end{tabular}

D7. [All] Do you consider yourself to be a....? (READ LIST) [RANDOMIZE ORDER OF 1-3; READ 4 ('Something else') LAST]

\begin{tabular}{|c|c|c|l|}
\hline $\begin{array}{c}\text { Base \% } \\
(\mathbf{n = 6 1 6 )}\end{array}$ & $\begin{array}{c}\text { AA/B \% } \\
(\mathbf{n = 2 0 0 )}\end{array}$ & $\begin{array}{c}\mathbf{H} / \mathbf{L} \% \\
(\mathbf{n = 2 0 4 )}\end{array}$ & \\
\hline 35 & 60 & 45 & Democrat \\
\hline 31 & 19 & 24 & Independent \\
\hline 27 & 11 & 17 & Republican \\
\hline 1 & 1 & 1 & Something else (specify) \\
\hline 3 & 6 & 7 & Don't know (VOL) \\
\hline 3 & 3 & 6 & Refused (VOL) \\
\hline
\end{tabular}

D8. [All] How would you characterize your political views? (READ LIST)

\begin{tabular}{|c|c|c|l|}
\hline $\begin{array}{c}\text { Base \% } \\
(\mathbf{n = 6 1 6 )}\end{array}$ & $\begin{array}{c}\text { AA/B \% } \\
(\mathbf{n = 2 0 0 )}\end{array}$ & $\begin{array}{c}\mathbf{H} / \mathbf{L} \% \\
(\mathbf{n = 2 0 4})\end{array}$ & \\
\hline 12 & 8 & 11 & Very conservative \\
\hline 21 & 15 & 15 & Somewhat conservative \\
\hline 31 & 31 & 21 & Moderate \\
\hline 16 & 17 & 22 & Somewhat liberal \\
\hline 13 & 17 & 14 & Very liberal \\
\hline 3 & 7 & 4 & None of the above/ something else (VOL) \\
\hline 3 & 5 & 9 & Not sure/ Don't know (VOL) \\
\hline 2 & 3 & 5 & Refused (VOL) \\
\hline
\end{tabular}

D9. [All] Do you own or rent your primary residence?

\begin{tabular}{|c|c|c|l|}
\hline $\begin{array}{c}\text { Base \% } \\
(\mathbf{n = 6 1 6 )}\end{array}$ & $\begin{array}{c}\text { AA/B \% } \\
(\mathbf{n = 2 0 0 )}\end{array}$ & $\begin{array}{c}\mathbf{H} / \mathbf{L} \% \\
(\mathbf{n = 2 0 4 )}\end{array}$ & \\
\hline 80 & 58 & 75 & Own \\
\hline 19 & 39 & 21 & Rent \\
\hline 2 & 2 & 2 & Something else [SPECIFY] \\
\hline 0 & 1 & 1 & Not sure/ Don't know (VOL) \\
\hline$<1$ & $<1$ & 1 & Refused (VOL) \\
\hline
\end{tabular}




\section{AARP}

D10. [All] We realize income is a private matter and so rather than ask you anything specific about your income, l'd like to ask you to please stop me when I get to the category that includes your household's income before taxes in 2020. Was it...? (READ LIST) [CONFIRM INCOME BREAKS]

\begin{tabular}{|c|c|c|l|}
\hline $\begin{array}{c}\text { Base \% } \\
(\mathbf{n}=\mathbf{6 1 6})\end{array}$ & $\begin{array}{c}\text { AA/B \% } \\
(\mathbf{n}=\mathbf{2 0 0})\end{array}$ & $\begin{array}{c}\mathbf{H} / \mathbf{L} \% \\
(\mathbf{n}=\mathbf{2 0 4})\end{array}$ & \\
\hline 2 & 12 & 2 & Less than $\$ 10,000$ \\
\hline 4 & 5 & 4 & $\$ 10,000$ to less than $\$ 20,000$ \\
\hline 9 & 10 & 6 & $\$ 20,000$ to less than $\$ 30,000$ \\
\hline 7 & 10 & 16 & $\$ 30,000$ to less than $\$ 40,000$ \\
\hline 9 & 8 & 11 & $\$ 40,000$ to less than $\$ 50,000$ \\
\hline 8 & 6 & 11 & $\$ 50,000$ to less than $\$ 60,000$ \\
\hline 10 & 13 & 9 & $\$ 60,000$ to less than $\$ 75,000$ \\
\hline 17 & 13 & 13 & $\$ 75,000$ to less than $\$ 100,000$ \\
\hline 11 & 8 & 9 & $\$ 100,000$ to less than $\$ 125,000$ \\
\hline 7 & 8 & 4 & $\$ 125,000$ to less than $\$ 150,000$ \\
\hline 5 & 5 & 5 & $\$ 150,000$ to less than $\$ 200,000$ \\
\hline 6 & 2 & 5 & $\$ 200,000$ or more \\
\hline 1 & 2 & 2 & Don't know (VOL) \\
\hline 5 & 1 & 5 & Refused (VOL) \\
\hline
\end{tabular}

D11. [All] We would like to learn more about retirement savings issues among the LGBTQ community. Do you identify as a member of the LGBTQ community?

\begin{tabular}{|c|c|c|l|}
\hline $\begin{array}{c}\text { Base \% } \\
(\mathbf{n = 6 1 6 )}\end{array}$ & $\begin{array}{c}\text { AA/B \% } \\
(\mathbf{n = 2 0 0 )}\end{array}$ & $\begin{array}{c}\mathbf{H} / \mathbf{L} \% \\
(\mathbf{n = 2 0 4 )}\end{array}$ & \\
\hline 6 & 8 & 15 & Yes \\
\hline 91 & 91 & 84 & No \\
\hline 3 & 1 & 2 & Prefer not to say (VOL) \\
\hline
\end{tabular}

